\title{
Séance du 31 mars 2011
}

Plateforme suisse des registres médicaux - Le Comité central de la FMH approuve le concept relatif à la future «Plateforme suisse des registres médicaux». Cette plateforme entend donner un aperçu des registres existants, standardiser la saisie des données et uniformiser la gestion des données.

Projet pilote «Evaluation et promotion des activités qualité» - Un groupe de travail nouvellement constitué évalue le rapport entre le coût et la charge de travail des activités en faveur de la qualité d'une part, et leur utilité d'autre part. Cette future grille d'évaluation doit être testée avec deux sociétés de discipline dans le cadre d'un projet pilote, puis combinée aux données issues du monitorage de la qualité.

\footnotetext{
Nouveau modèle de rémunération de la remise de médicaments - Avec sa proposition d'une remise de médicaments exempte de marge, la FMH répond par une solution claire et réalisable aux perpétuelles allégations selon lesquelles la propharmacie crée de faux incitatifs et que les médecins ne devraient pas tenir de «commerce de médicaments». La rémunération doit à l'avenir s'effectuer par le biais d'une prestation médicale (PM) et d'une prestation technique (PT) dans le TARMED. La transition ne doit pas avoir d'incidence sur les coûts. La première ronde de négociations avec santésuisse a eu lieu à la mi-avril 2011.
}

Efficacité, adéquation, économicité - Le CC de la FMH accepte que son groupe de travail EAE développe avec santésuisse une stratégie commune visant à créer de nouvelles bases pour les procédures EAE. Une initiative parlementaire à ce sujet devrait être examinée lors de la session d'été; une modification de la loi pourrait entrer en vigueur au $1^{\text {er }}$ janvier 2012.

Enquête sur la qualité de la formation postgraduée - Le CC prend connaissance du fait que, comme l'année dernière, les médecins-assistants seront interrogés sur la qualité de la formation postgraduée [1]. Un atelier se tiendra en novembre 2012 pour déterminer la manière dont cette enquête devrait être conduite dans le futur.

Recommandations en matière $d^{\prime}$ IST - En décembre 2010, l'OFSP a présenté le nouveau «Programme national VIH et autres infections sexuellement transmissibles 2011-2017». La campagne a débuté fin mars. Il faut s'attendre à ce que les personnes sensibilisées s'adressent toujours plus aux médecins de premier recours, raison pour laquelle l'OFSP a élaboré à l'intention de ces derniers des recommandations [2] concernant le diagnostic et le traitement des IST, sur lesquelles les sociétés de discipline et la FMH ont également pris position. 